\title{
Transmission Electron Microscopy of Lipid Vesicles in Liquid Cells
}

\author{
C. M. Brown ${ }^{1}$, K. L. Klein ${ }^{1,2}$, D. V. Krogstad ${ }^{3}$, G. A. Myers ${ }^{1}$, A. A. Herzing ${ }^{1}$ \\ ${ }^{1}$ Materials Measurement Science Division, NIST, Gaithersburg, MD 20899, USA \\ 2 Department of Mechanical Engineering, University of the District of Columbia, Washington, DC \\ 20008, USA \\ ${ }^{3}$ Biosystems and Biomaterials Division, NIST, Gaithersburg, MD 20899, USA
}

Liquid cell transmission electron microscopy (TEM) shows promise for enabling nanometer-scale imaging of fluid-hydrated biological material by enclosing specimens within windowed devices [1]. However, the thickness of the liquid cell reduces the signal-to-noise ratios of acquired images, a particular problem for researchers imaging biological specimens. Thus investigators typically image biological specimens labeled with high atomic number materials [2] or with the aid of negative stains [3]. Nevertheless, it is possible to avoid exogenous contrast agents by imaging specimens in thin fluid layers while using energy-filtered methods to reduce collection of inelastically scattered electrons from water and window material. Here, we report on acquisition of liquid cell images of unstained dioleoylglycerophosphatidylcholine (DOPC) lipid vesicles using an energy-filtered TEM (EFTEM) equipped with an imaging energy filter. Liquid cell operating parameters and EFTEM focal conditions that allow for visualization of unstained fluid hydrated vesicles have been determined and will be discussed.

50-nm-thick-windowed SiNx devices were prepared in a clean room environment using high purity solvents followed by a brief plasma clean. DOPC lipid vesicles were synthesized by extruding $1 \mathrm{mg}$ DOPC/750 $\mu \mathrm{L}$ deionized water through a $100 \mathrm{~nm}$ pore size track-etch filter, synthesizing uni-lamellar and multi-lamellar vesicles which were $50-150 \mathrm{~nm}$ in diameter. $3 \mu \mathrm{L}$ of vesicle solution was drop cast on the windowed device. A second windowed device with a $150 \mathrm{~nm}$ spacer enclosed the sample so that the two windows aligned orthogonally to form a liquid cell. Using a syringe pump, water was pumped at $<50 \mu \mathrm{L} / \mathrm{hr}$ through the holder and around the liquid cell and sample to remove any air pockets from the system. Once the holder was introduced into the TEM, the pump flow rate was decreased or stopped, reducing the risk of removing the sample from the windowed region of the cell and minimizing outward bowing of the SiNx windows in vacuum. Bright field, zero-loss EFTEM images were acquire using a field emission TEM operated at $300 \mathrm{kV}$ with a $10-\mathrm{eV}$ wide energy slit and a $5.4 \mathrm{mrad}$ objective aperture. Lipid vesicles were visualized at $72,500 \times-120,000 \times$ magnification with frame rates ranging from 0.25 to $1 \mathrm{~s}$ per frame.

Due to the vacuum in the TEM, the SiNx windows of the liquid cell bow outward as previously described [4]. Bowing of the windows was quantified with a mass-thickness image of a liquid cell, as shown in Fig. 1a. Images of vesicles were acquired within $<1 \mu \mathrm{m}$ lateral distance from the corners of the windowed liquid cell. The vesicle concentration in solution ensured samples could be imaged at these locations. EFTEM images of fluid hydrated lipid vesicles were acquired and show a smooth spherical appearance, as shown in Fig. 1b. A focal series of a lipid vesicle was acquired, as shown in Fig. 2. This data shows that contrast in acquired vesicle images increases at significant defocus, similar to typical cryo-TEM imaging conditions. The motion of particles around the vesicle indicates it is fluid hydrated. 
We will further discuss aspects of experimental setup, instrument operation, avenues to improve the image quality of liquid cell specimens, and the feasibility of EFTEM imaging of unstained biological specimens in a hydrated fluid environment [5].

\section{References:}

[1] KL Klein et al, Journal of Microscopy 242 (2011), p. 117.

[2] N de Jonge et al, Proceedings of the National Academy of Sciences 106 (2009) p. 2159. [3] BL Gilmore et al, Lab on a Chip 13 (2013), p. 216.

[4] KL Klein and IM Anderson, Microscopy and Microanalysis (2012), p 1154.

[5] CMB, DVK and GAM acknowledge support from the National Research Council Postdoctoral Research Associateship Program.

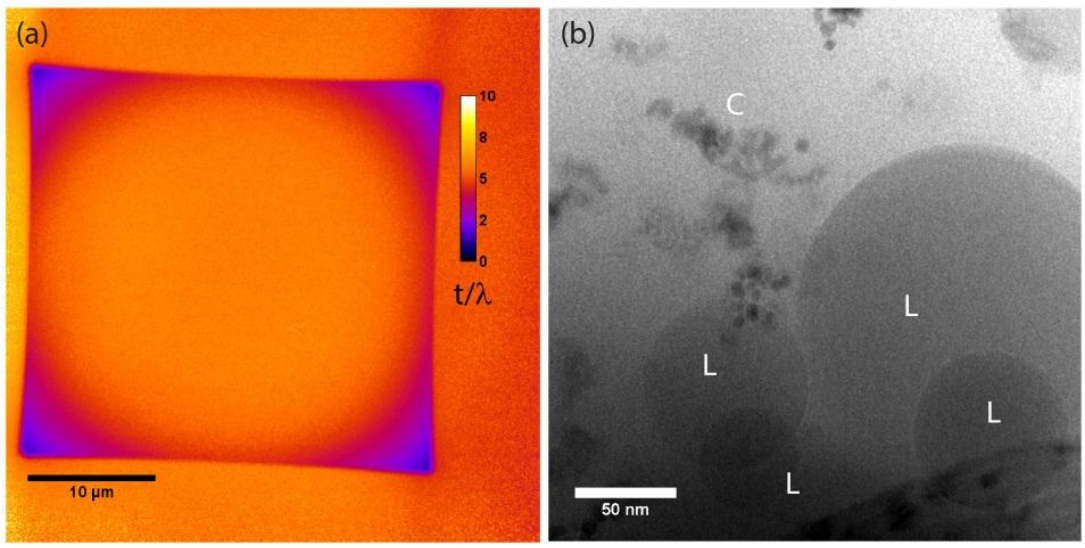

Figure 1. (a) Mass-thickness $(\mathrm{t} / \lambda)$ image of the $\mathrm{SiNx}$ windowed liquid cell filled with water. The ratio of thickness $(t)$ to inelastic scattering mean-free-path $(\lambda)$ is determined by calculating the ratio of the EFTEM unfiltered $\left(\mathrm{I}_{\mathrm{t}}\right)$ to zero-loss $\left(\mathrm{I}_{0}\right)$ images according to $\mathrm{t} / 1=\ln \left(\mathrm{I}_{\mathrm{t}} / \mathrm{I}_{\mathrm{o}}\right)$. (b) EFTEM image of lipid vesicles (L) and unidentified particles $(\mathrm{C})$ near corner of windowed cell.

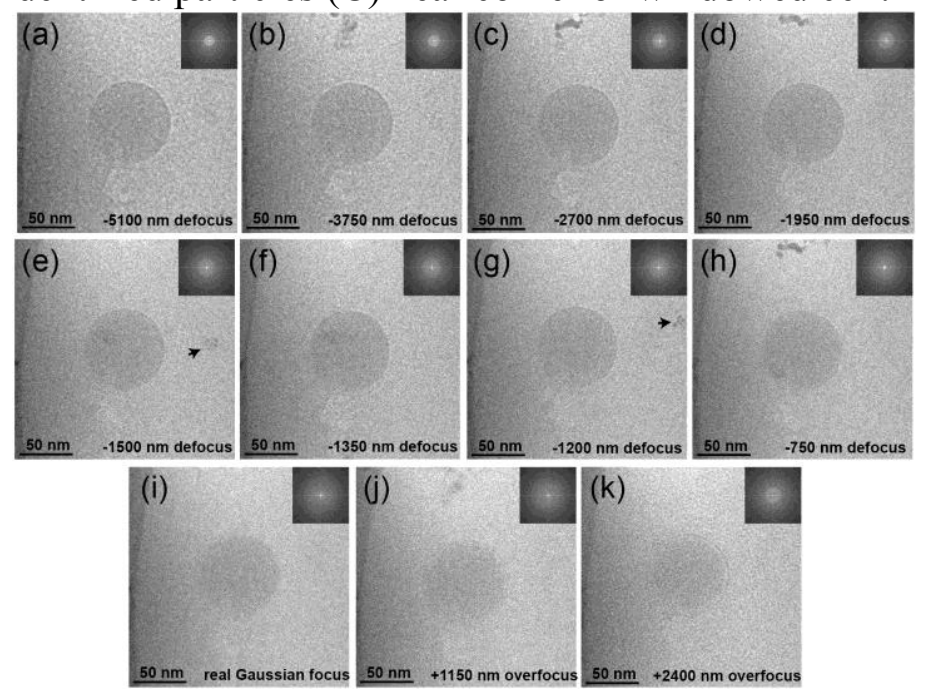

Figure 2. Focal series of lipid vesicle in liquid cell. Images show focal conditions from $-5100 \mathrm{~nm}$ defocus (a) to $+2400 \mathrm{~nm}$ overfocus $(\mathrm{k})$. The focal series was centered on the $-1350 \mathrm{~nm}$ defocus image (f) and acquired in the following sequence: $\mathrm{f}, \mathrm{g}, \mathrm{e}, \mathrm{h}, \mathrm{d}, \mathrm{i}, \mathrm{c}, \mathrm{j}, \mathrm{b}, \mathrm{k}$, a. Arrows indicate motion of particles in solution throughout the focal series. 